\title{
Reduced Complexity Detection for Ricean MIMO Channels Based on Condition Number Thresholding
}

\author{
Michail Matthaiou*, David I. Laurenson*, and Cheng-Xiang Wang ${ }^{\dagger}$ \\ *Institute for Digital Communications, Joint Research Institute for Signal and Image Processing, \\ School of Engineering and Electronics, The University of Edinburgh, \\ Mayfield Road, EH9 3JL, Edinburgh, U.K. \\ Email: \{M.Matthaiou, Dave.Laurenson\}@ed.ac.uk \\ $\dagger$ Joint Research Institute for Signal and Image Processing, \\ School of Engineering and Physical Sciences, Heriot-Watt University, \\ EH14 4AS, Edinburgh, U.K. \\ Email: Cheng-Xiang.Wang@hw.ac.uk
}

\begin{abstract}
In this paper, a novel adaptive detection scheme for the case of Multiple-Input Multiple-Output (MIMO) Ricean channels with two transmit and receive antenna elements is presented. Our prime aim is to reduce the extensive complexity of Maximum-Likelihood (ML) detectors by developing an adaptive scheme which switches between a ML and a much simpler ZeroForcing ( $\mathrm{ZF})$ detector depending on the instantaneous spatial conditions. The kernel of the adaptive detector (AD) is a harddecision criterion based on the condition number of the MIMO correlation matrix. It is demonstrated that the proposed scheme offers a remarkable reduction in terms of complexity along with a satisfactory performance when specifically designed antenna arrays are employed.
\end{abstract}

\section{INTRODUCTION}

The use of MIMO technology has become the new hot topic in wireless communications since the breakthrough works of Telatar [1] and Foschini [2] revealed the great advantages of employing multiple antennas at both the transmitter (Tx) and receiver $(\mathrm{Rx})$. This technology can potentially enhance the reliability and speed of current and future wireless systems such as wireless local area networks (WLANs) or fourth generation cellular systems (4G). However, when it comes down to the feasibility of MIMO systems, a number of practical issues arises with the most prominent being the overall implementation complexity and cost.

Undoubtedly, the detection stage may be regarded as one of the most significant aspects in real-time applications. At this point, we recall that the optimal detector for spatial multiplexing (SM) MIMO systems is the ML detector which minimizes the error probability when all data vectors are equally likely but, at the same time, it is computationally prohibitive [3]. One way to alleviate this excessive complexity is to settle for sphere decoding techniques, such as the Finke-Post algorithm proposed in [4], whose complexity, under certain assumptions, is polynomial in the problem size. In [5], it was shown that when the Signal-to-Noise ratio (SNR) is high, the expected number of operations required by the sphere decoder is roughly cubic in the number of transmit antennas for a small problem size. However, the authors in [6] proved that for any arbitrarily fixed SNR, the overall complexity of sphere decoders does not grow as a polynomial function of the problem size but as an exponential function instead. What's more, when illconditioned channels occur, the computational complexity of sphere decoding schemes increases to a significant extent [7]. On the other hand, different suboptimal techniques exist which span from the linear ZF detector to nonlinear techniques such as Ordered Successive Interference Cancellation (OSIC) [8]; the former is the simplest detection technique but causes a systematic performance degradation and further is unable to exploit all of the available diversity. Its main disadvantage lies in its poor performance when channels with large condition numbers occur.

The previous discussion implies that an $\mathrm{AD}$ which could switch between a ML and a ZF scheme, depending on the instantaneous channel conditions, is of paramount interest since it will allow the efficient development of MIMO systems. In this paper, an $\mathrm{AD}$ is devised for the general case of Ricean MIMO channels where a dominant Line-of-Sight (LoS) component or a specular wavefront impinges on the receive array; we are particularly interested in the practical case of dual $^{1}$ Ricean MIMO systems. A similar concept of adaptive MIMO transmission has been investigated by various research groups during recent years (the interested readers are referred to [9]-[11] among others) and essentially goes back to the fundamental diversity-multiplexing tradeoff [12]. To the best of the authors' knowledge, the first MIMO adaptive scheme for the receive side was recently presented in [13]. The authors therein, however, considered only the common case of Rayleigh channels and hence the formulation of a generalized framework is infeasible; the proposed scheme relaxes the constraint of Rayleigh fading to account for the commonly experienced Ricean propagation and, consequently, it includes the model of [13] as a special case.

The hard-decision criterion for the adaptive switching relies on the condition number distribution whose exact determination requires knowledge of the theory of non-central complex

\footnotetext{
${ }^{1}$ Throughout the paper, the term dual will stand for MIMO systems with two transmit and two receive antenna elements.
} 
Wishart matrices. On these grounds, we firstly derive a novel closed-form formula for the cumulative distribution function (CDF) of the MIMO condition number which is validated via Monte-Carlo simulations. It will also be shown that the $\mathrm{AD}$ preserves the robustness of the ML detector as well as the simplicity of the ZF detector and therefore can be easily implemented within most MIMO testbeds.

The remainder of the paper is organized as follows: In Section II, the preliminaries of the theory of Wishart matrices are outlined and a closed-form expression for the condition number CDF distribution is derived. In Section III, the underlying MIMO Ricean channel model used throughout the paper is presented. In Section IV, we briefly address the main characteristics of ZF and ML detections and subsequently those of the proposed adaptive scheme. In Section V, the validity of the analytical formula is tested and at a next stage we assess the performance of the AD for two different LoS geometrical configurations. Finally, Section VI concludes the paper and summarizes the key findings.

A note on notation: We use upper and lower case boldface to denote matrices and vectors, respectively while the symbol $\mathbb{C}$ denote the set of complex-valued numbers. The nomenclature $\sim \mathcal{C N}(\mathbf{X}, \mathbf{Y})$ stands for a complex normally distributed matrix with mean $\mathbf{X}$ and covariance $\mathbf{Y}$. An $(n \times n)$ identity matrix is expressed as $\mathbf{I}_{n}$ while the all-zeros $(n \times m)$ matrix as $\mathbf{0}_{n \times m}$. The symbols $(\cdot)^{H}$ and $(\cdot)^{-1}$ correspond to Hermitian transposition and matrix inversion, respectively whereas $\otimes$ is the Kronecker product. Finally, $\operatorname{det}(\cdot)$ returns the determinant of a matrix.

\section{Non-Central Wishart Matrices and Condition Number Distribution}

As was previously mentioned, we are particularly interested in dual non-central Wishart matrices. In such a case, a $(2 \times 2)$ complex normal random matrix $\mathbf{H}$ is considered which is distributed according to $\mathbf{H} \sim \mathcal{C N}\left(\mathbf{M}, \boldsymbol{\Sigma} \otimes \mathbf{I}_{2}\right)$. In general, $\mathbf{M} \neq \mathbf{0}_{2 \times 2}$ whereas $\boldsymbol{\Sigma}=\sigma^{2} \mathbf{I}_{2}$ is the correlation matrix containing the variances $\sigma^{2}$ of the entries of $\mathbf{H}$ on its main diagonal. The so-called MIMO correlation matrix is defined as $\mathbf{W}=\mathbf{H H}^{H}$ and is said to follow the complex noncentral Wishart distribution with two degrees of freedom and non-centrality matrix $\boldsymbol{\Omega}=\boldsymbol{\Sigma}^{-1} \mathbf{M} \mathbf{M}^{H}$, commonly denoted as $\mathbf{W} \sim \mathcal{C W}_{2}(2, \boldsymbol{\Sigma}, \boldsymbol{\Omega})$. It should be emphasized that if $\mathbf{M}=\mathbf{0}_{2 \times 2}$, so that $\boldsymbol{\Omega}=\mathbf{0}_{2 \times 2}$, a complex central Wishart matrix is eventually obtained, i.e. $\mathbf{W} \sim \mathcal{C W}_{2}(2, \boldsymbol{\Sigma})$.

We now consider a scaled version of $\mathbf{W}$, that is $\widetilde{\mathbf{W}}=$ $\boldsymbol{\Sigma}^{-1} \mathbf{W}$. Since $\widetilde{\mathbf{W}}$ is a $(2 \times 2)$ Hermitian matrix, it has two real ordered eigenvalues $\left(w_{1}>w_{2}>0\right)$ where $w_{1}$ is the largest and $w_{2}$ the smallest eigenvalue, respectively; the joint eigenvalue $\operatorname{PDF} f\left(w_{1}, w_{2}\right)$ is given by [14]

$f\left(w_{1}, w_{2}\right)=\exp \left[-\sum_{i=1}^{2}\left(\lambda_{i}+w_{i}\right)\right]{ }_{0} \widetilde{F}_{1}(2 ; \boldsymbol{\lambda}, \mathbf{w})\left(w_{1}-w_{2}\right)^{2}$

where $\boldsymbol{\lambda}=\left(\lambda_{1}, \lambda_{2}\right)$ contains the distinct real ordered eigenvalues of $\boldsymbol{\Omega}$ and, in turn, $\mathbf{w}=\left(w_{1}, w_{2}\right)$; furthermore, ${ }_{0} \widetilde{F}_{1}(. ; .,$.$) is$ the complex hypergeometric function of two matrix arguments. A convenient version of ${ }_{0} \widetilde{F}_{1}(2 ; \boldsymbol{\lambda}, \mathbf{w})$ for the dual case was given by Gross and Richards [15] as

$$
{ }_{0} \widetilde{F}_{1}(2 ; \boldsymbol{\lambda}, \mathbf{w})=\frac{\operatorname{det}\left({ }_{0} F_{1}\left(1 ; w_{i} \lambda_{j}\right)\right)}{\left(\lambda_{1}-\lambda_{2}\right)\left(w_{1}-w_{2}\right)}
$$

with $I_{q}(\cdot)$ denoting the $q$-th order modified Bessel function of the first kind and ${ }_{0} F_{1}(s+1 ; x)$ is the hypergeometric function [16]

$$
{ }_{0} F_{1}(s+1 ; x)=s ! x^{-s / 2} I_{s}(2 \sqrt{x}) .
$$

From a mathematical point of view, the condition number $z$ is defined as the ratio of the largest to the smallest eigenvalue and therefore

$$
z=\frac{w_{1}}{w_{2}} \geq 1 .
$$

In [17], the authors showed that the probability density function (PDF) of $z, f_{z}(z)$, can be written as a weighted summation of polynomials according to (5), shown at the top of the next page. In (5), $\Gamma(n)$ denotes the Gamma function which, for the case of an integer index, can be rewritten as $\Gamma(n)=(n-1)$ !. The corresponding CDF of $z$ is then directly written as

$$
F_{z}(x)=\int_{1}^{x} f_{z}(z) d z
$$

or, equivalently

$$
F_{z}(x)=\int_{0}^{x} f_{z}(z) d z-\int_{0}^{1} f_{z}(z) d z .
$$

By substituting (5) into (7) and taking into account the Dominated Convergence Theorem which suggests that the differentiation and integration can be interchanged we readily obtain (8). For the integrals involved in (8), a closed-form solution is available as [16, Eq. (3.194)]

$$
\int_{0}^{u} \frac{t^{\mu}}{(1+b t)^{\nu}} d t=\frac{u^{\mu+1}}{\mu+1}{ }_{2} F_{1}(\nu, \mu+1 ; \mu+2 ;-b u)
$$

where ${ }_{2} F_{1}(\alpha, \beta ; \gamma ; u)$ is the classical Gaussian hypergeometric function defined in [16, Eq. (9.14)]. We can finally write the CDF of the condition number according to (9), where

$$
\begin{aligned}
I_{1}^{k, n}(x) & =\left(\frac{x^{k+2}}{k+2}\right){ }_{2} F_{1}(k+n+3, k+2 ; k+3 ;-x) \\
& -\left(\frac{1}{k+2}\right){ }_{2} F_{1}(k+n+3, k+2 ; k+3 ;-1)
\end{aligned}
$$

and

$$
\begin{aligned}
I_{2}^{k, n}(x) & =\left(\frac{x^{k+1}}{k+1}\right){ }_{2} F_{1}(k+n+3, k+1 ; k+2 ;-x) \\
& -\left(\frac{1}{k+1}\right){ }_{2} F_{1}(k+n+3, k+1 ; k+2 ;-1) .
\end{aligned}
$$

Clearly, we have expressed the condition number distribution as a weighted summation of Gaussian hypergeometric functions which can be efficiently evaluated and easily programmed. 


$$
\begin{gathered}
f_{z}(z)=\frac{e^{-\left(\lambda_{1}+\lambda_{2}\right)}(z-1)}{\lambda_{1}-\lambda_{2}} \sum_{k=0}^{\infty} \sum_{n=0}^{\infty} \frac{\Gamma(k+n+3) z^{k}}{(k ! n !)^{2}(z+1)^{k+n+3}}\left[\lambda_{1}^{k} \lambda_{2}^{n}-\lambda_{2}^{k} \lambda_{1}^{n}\right] \\
F_{z}(x)=\frac{e^{-\left(\lambda_{1}+\lambda_{2}\right)}}{\lambda_{1}-\lambda_{2}} \sum_{k=0}^{\infty} \sum_{n=0}^{\infty} \frac{\Gamma(k+n+3)}{(k ! n !)^{2}}\left[\lambda_{1}^{k} \lambda_{2}^{n}-\lambda_{2}^{k} \lambda_{1}^{n}\right] \\
\times\left\{\int_{0}^{x} \frac{z^{k+1}}{(z+1)^{k+n+3}}-\int_{0}^{1} \frac{z^{k+1}}{(z+1)^{k+n+3}}-\int_{0}^{x} \frac{z^{k}}{(z+1)^{k+n+3}}+\int_{0}^{1} \frac{z^{k}}{(z+1)^{k+n+3}}\right\} \\
F_{z}(x)=\frac{e^{-\left(\lambda_{1}+\lambda_{2}\right)}}{\lambda_{1}-\lambda_{2}} \sum_{k=0}^{\infty} \sum_{n=0}^{\infty} \frac{\Gamma(k+n+3)}{(k ! n !)^{2}}\left[\lambda_{1}^{k} \lambda_{2}^{n}-\lambda_{2}^{k} \lambda_{1}^{n}\right]\left\{I_{1}^{k, n}(x)-I_{2}^{k, n}(x)\right\}
\end{gathered}
$$

\section{MIMO CHANNEL MODEL}

In this section, the underlying MIMO channel model is discussed for the case of LoS propagation. We consider a memoryless, flat-fading MIMO system equipped with two elements at both the Tx and the Rx. The complex input-output relationship can be written for the discrete case as

$$
\mathbf{y}=\mathbf{H x}+\mathbf{n}
$$

where $\mathbf{x} \in \mathbb{C}^{2 \times 1}$ is the transmitted signal vector, $\mathbf{y} \in \mathbb{C}^{2 \times 1}$ is the noise-corrupted received signal and $\mathbf{n} \in \mathbb{C}^{2 \times 1}$ corresponds to the additive noise plus interference term. Moreover, the term $\mathbf{H} \in \mathbb{C}^{2 \times 2}$ is referred to as the channel transfer function matrix and contains the complex responses between all antenna pairs.

In the case of Ricean fading, the channel matrix consists of a spatially deterministic specular component $\mathbf{H}_{\mathrm{L}}$ and a randomly distributed component $\mathbf{H}_{\mathrm{W}}$ which accounts for the scattered signals. Then, the channel model reads as [18]

$$
\mathbf{H}=\sqrt{\frac{K}{K+1}} \mathbf{H}_{\mathrm{L}}+\sqrt{\frac{1}{K+1}} \mathbf{H}_{\mathrm{W}}
$$

where $K$ denotes the Ricean $K$-factor expressing the ratio of the free-space signal power to the power of the scattered waves. The entries of $\mathbf{H}_{\mathrm{W}}$ are assumed to be independent and identically distributed (i.i.d.) circular symmetric complex Gaussian variables with zero mean and unity variance so that their amplitudes follow the well-known Rayleigh distribution. As far as the LoS component $\mathbf{H}_{\mathrm{L}}$ is concerned, when the distance between the $\mathrm{Tx}$ and the $\mathrm{Rx}$ is small or the array size is large, its entries represent spherical wavefronts in the near-field region. Without loss of generality, we assume isotropic radiators and the complex responses are of the form $e^{-j k d_{m, n}} / d_{m, n}$, where $k=2 \pi / \lambda$ is the wavenumber corresponding to the carrier wavelength $\lambda$ and $d_{m, n}$ is the distance between a receive element $m \in\{1,2\}$ and a transmit element $n \in\{1,2\}$.

Regarding the statistical characteristics of $\mathbf{H}$, it can be inferred that $\mathbf{M}=\sqrt{\frac{K}{K+1}} \mathbf{H}_{\mathrm{L}}$ while $\boldsymbol{\Sigma}=\frac{1}{K+1} \mathbf{I}_{2}$. Then, it is trivial to show that the Wishart matrix $\mathbf{W}=\mathbf{H} \mathbf{H}^{H}$ follows the distribution $\mathbf{W} \sim \mathcal{C} \mathcal{W}_{2}\left(2, \frac{1}{K+1} \mathbf{I}_{2}, K \mathbf{H}_{\mathrm{L}} \mathbf{H}_{\mathrm{L}}^{H}\right)$ and in turn the associated LoS version of interest $\widetilde{\mathbf{W}}=(K+1) \mathbf{W}$.

\section{Detection Schemes For SM Systems}

In this section we review the two reference detection schemes, namely ZF and ML detectors, and explore the concept of the novel adaptive detection strategy. All the following investigations are based on a SM-MIMO transmission scheme, such as the widely employed V-BLAST [8], in which the data is divided into a number of $N_{t}$ blocks (equal to the number of transmit elements) that are then simultaneously emitted. At the $\mathrm{Rx}$, the main goal is to differentiate the data blocks originating from each of the transmit elements so that the transmitted signals are efficiently recovered.

\section{A. ZF detection}

The simplest linear MIMO detector is the $\mathrm{ZF}$ receiver, where the received signal vector $\mathbf{y}$ is multiplied by the MoorePenrose pseudoinverse $\mathbf{H}^{\dagger}$ of the channel matrix $\mathbf{H}$ to obtain an estimated transmit signal vector $\hat{\mathbf{x}}_{\mathrm{ZF}}$ as follows

$$
\hat{\mathbf{x}}_{\mathrm{ZF}}=\mathbf{H}^{\dagger} \mathbf{H} \mathbf{x}+\mathbf{H}^{\dagger} \mathbf{n} \text {. }
$$

The computational complexity of ZF includes an exhaustive search through the $Q$ symbols in the constellation of the modulation technique for $N_{t}$ times and thus it is of the order of $O\left(Q N_{t}\right)$. However, the low complexity of the $\mathrm{ZF}$ receiver comes at the expense of noise amplification which induces irreducible errors. In fact, as the number of transmit and receive antennas grows with no bound, the noise amplification tends to infinity [19].

\section{B. $M L$ detection}

On the other end, the optimal ML detector resides which remains robust and yields the best performance among all detection techniques [3]. Assuming equally likely, temporally uncoded transmit symbols, this receiver chooses the vector $\mathbf{t}$ that solves the following expression

$$
\hat{\mathbf{x}}_{\mathrm{ML}}=\arg \min _{\mathbf{t}}\|\mathbf{y}-\mathbf{H} \mathbf{t}\|_{F}^{2}
$$


The optimization is performed through an exhaustive search over all possible vector symbols. This implies that the complexity of the ML detector grows exponentially with the number of transmit antennas i.e. $O\left(Q^{N_{t}}\right)$, making the scheme infeasible for large antenna setups and constellation sizes.

\section{Adaptive detection}

Given the deficiencies of both detection strategies, we herein propose a novel detector which can adaptively switch between them in order to enhance the error performance and minimize the computational cost. The AD uses $\mathrm{ZF}$ when the condition number is below a predefined threshold defined and ML detection otherwise. The key notion is to employ the ZF detector only for well-conditioned channels (low condition numbers) and let the ML deal with the ill-conditioned channels (high condition numbers). We can then write

$$
\hat{\mathbf{x}}_{\mathrm{AD}}= \begin{cases}\hat{\mathbf{x}}_{\mathrm{ZF}} & \text { if } z \leq \kappa \\ \hat{\mathbf{x}}_{\mathrm{ML}} & \text { if } z>\kappa .\end{cases}
$$

The threshold $\kappa$ affects the complexity of the proposed scheme; for $\kappa=1$ we get $\hat{\mathbf{x}}_{\mathrm{AD}}=\hat{\mathbf{x}}_{\mathrm{ML}}$ and complexity equals that of ML detection whereas for $\kappa \rightarrow \infty$ we have $\hat{\mathbf{x}}_{\mathrm{AD}}=\hat{\mathbf{x}}_{\mathrm{ZF}}$. In general, the probability of $\mathrm{ZF}$ calls is Prob $\{z \leq \kappa\} \equiv F_{z}(\kappa)=p_{\kappa}$ and therefore the average AD complexity $g$ becomes

$$
g=\underbrace{p_{\kappa} Q N_{t}}_{\text {ZF calls }}+\underbrace{\left(1-p_{\kappa}\right) Q^{N_{t}}}_{\text {ML calls }}, \quad 0 \leq p_{\kappa} \leq 1 .
$$

The percentage of complexity reduction, compared to that of the ML detector, is

$$
\frac{Q^{N_{t}}-g}{Q^{N_{t}}}=p_{\kappa}\left(1-\frac{N_{t}}{Q^{N_{t}-1}}\right) .
$$

Evidently, the reduction is more pronounced for greater sizes of the symbol alphabet and a higher number of ZF calls.

\section{Simulation Results}

In this section, the theoretical analysis presented in Section II is validated through a set of simulations and subsequently the link-level performance of the $\mathrm{AD}$ is assessed in terms of complexity and bit-error-rate (BER). In order to get a deeper insight, we explore two different geometrical models for the LoS component.

In particular, the first model belongs in the family of highrank (HR) configurations which, contrary to the common belief, can deliver high MIMO capacities in the presence of strong LoS components (large $K$-factors). This is achieved by appropriate positioning of the antenna elements at both ends so that subchannel orthogonality is attained, or, the eigenvalues of $\Omega$ become equal. In such a case though, the $\left(\lambda_{1}-\lambda_{2}\right)$ term in the denominator of (2) becomes zero making the analysis invalid (division by zero). In this light, we consider a suboptimum HR LoS channel model in order to guarantee that $\lambda_{1} \neq \lambda_{2}$; the LoS matrix component then reads [17]

$$
\mathbf{H}_{\mathrm{L}}=\left[\begin{array}{cc}
0.8384+j 0.5451 & 0.9411+j 0.3380 \\
-0.5123-j 0.8588 & 0.8384+j 0.5451
\end{array}\right]
$$

Assuming a Ricean- $K$ factor of $5 \mathrm{~dB}$, the eigenvalues of $\Omega$ can be easily computed and thereafter concatenated into the vector $\boldsymbol{\lambda}=(7.0336,5.6155)$. The second model is a conventional architecture whose deterministic matrix reads

$$
\mathbf{H}_{\mathrm{L}}^{\prime}=\left[\begin{array}{cc}
0.8384+j 0.5451 & 0.9411+j 0.3380 \\
-0.5123-j 0.8588 & 0.8384+j 0.5451
\end{array}\right]
$$

and, likewise, $\boldsymbol{\lambda}^{\prime}=(12.649,0.0001)$. For both geometrical models under investigation, 50,000 random Monte-Carlo realizations of the channel matrix $\mathbf{H}$ were generated according to (14). In Fig. 1, the simulated CDF curves of the condition number are overlaid with the analytical results returned by (9).

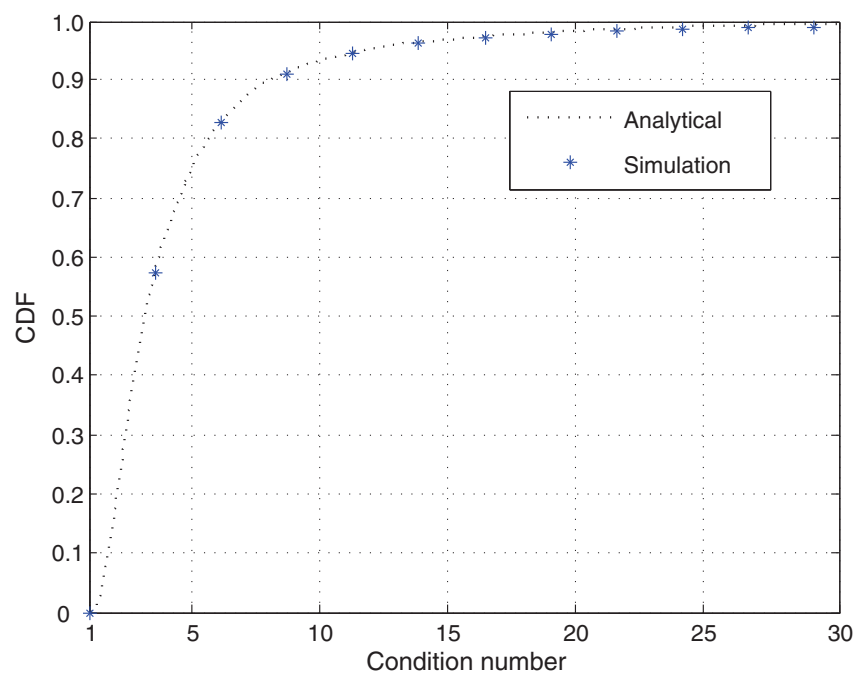

(a) HR LoS channel.

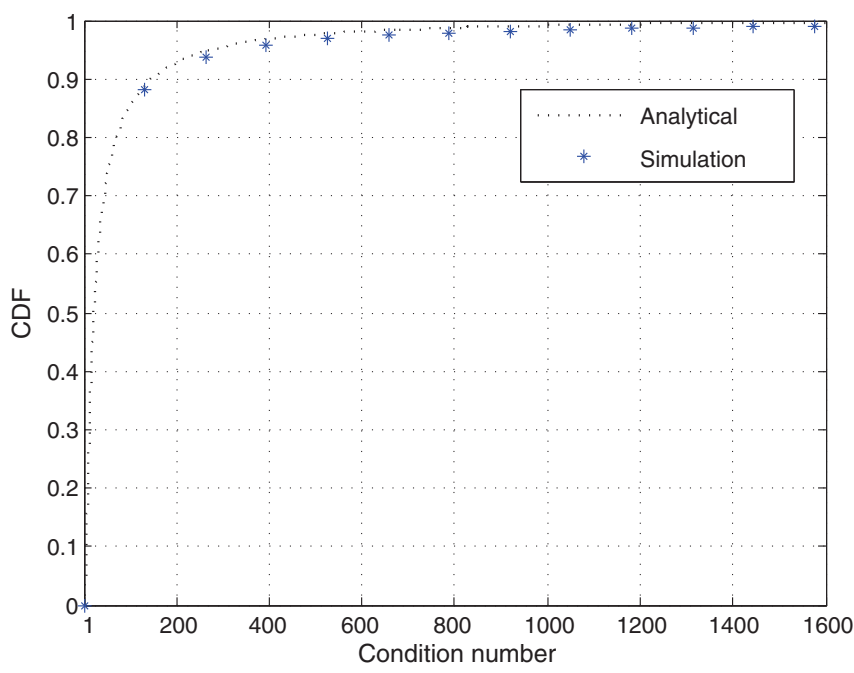

(b) LR LoS channel.

Fig. 1. Simulated and analytical CDFs of the condition number $z$ of two Ricean MIMO channel models $(K=5 \mathrm{~dB})$.

The match between the simulated and theoretical results is excellent in both cases, thereby indicating the validity of the derived formulae. We also point out that for the HR model a significant percentage of realizations is close to unity 
(well-conditioned channels) while, in contrary, the LR model systematically yields a rather high condition number.

The next stage of our investigation comprises the assessment of the AD advantages assuming a 16-QAM modulation type. In [17], we found that when $\kappa \leq 6.46$ the instantaneous capacity of the HR channel is greater than the ergodic capacity of a common $(2 \times 2)$ i.i.d. Rayleigh channel and hence we have adopted a threshold of $\kappa=5$ as a reasonable indicator of the channel rank and multipath richness. In Fig. 2, the BER curves are depicted for three different detection schemes, namely $\mathrm{ZF}$, ML and the proposed $\mathrm{AD}$. As anticipated, higher $K$ factors lead to significant performance enhancement for arrays designed following the suboptimum HR model. On the other hand, for low values of $K$-factor (below $0 \mathrm{~dB}$ ) the channel approaches the i.i.d. Rayleigh channel characteristics which corresponds to a rich-scattering environment; in this region, the BER curves are approximately identical in both systems and the benefits of LoS-optimized arrays are minimized. In the limit $(K \rightarrow \infty \mathrm{dB})$ the LoS component vanishes and we end up with a pure i.i.d Rayleigh channel where all multipath components have equal amplitudes.

In order to get a better understanding, the main $A D$ performance characteristics are tabulated in Table I where the radically different trends of the two configurations are readily observable. More specifically, the application of the detection scheme has a noticeable impact on the HR channel; in fact, a significant percentage of ZF calls occur for all values of the $K$-factor with a consequent complexity reduction of up to $83.35 \%$. We also observe a steady increase of $p_{\kappa}$ as $K$ gets higher since the channel becomes full rank and delivers two approximately equal eigenvalues. In the LR case $p_{\kappa}$ is always below $29.43 \%$ and further is inversely proportional to the $K$ factor. This phenomenon can be attributed to the ill-condition of conventional architectures which degenerate eventually into a rank-one channel due to the linear dependence of the LoS rays' phases (higher spatial correlation). Consequently, for $K \geq 5 \mathrm{~dB}$ the number of $\mathrm{ZF}$ calls is too low to exploit the adaptivity benefits and a complexity identical to that of a ML detector inevitably takes place.

\section{CONCLUSION}

In the present contribution, the potential of developing an $\mathrm{AD}$ relying on the condition number of the MIMO correlation matrix, has been investigated. On this basis, we firstly derived a closed-form formula for the CDF of the condition number of dual non-central complex Wishart matrices as a weighted summation of Gaussian hypergeometric functions. The analytical CDF expression was firstly validated through extensive Monte-Carlo simulations where it was clearly demonstrated that the match between theory and simulation is very good. At a next stage, the distribution of the condition number was used to construct the hard-decision criterion of the proposed $\mathrm{AD}$ which is appealing not only from a theoretical but also from a practical perspective; most importantly, the dramatic decrease in terms of complexity, compared to the sophisticated ML detector, makes the model applicable to the majority of

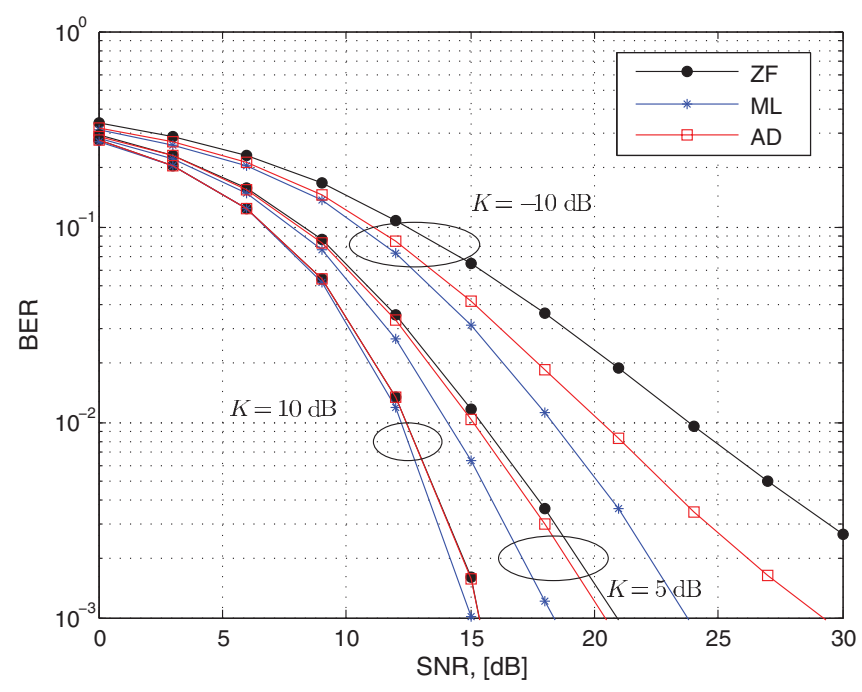

(a) Suboptimum HR LoS channel.

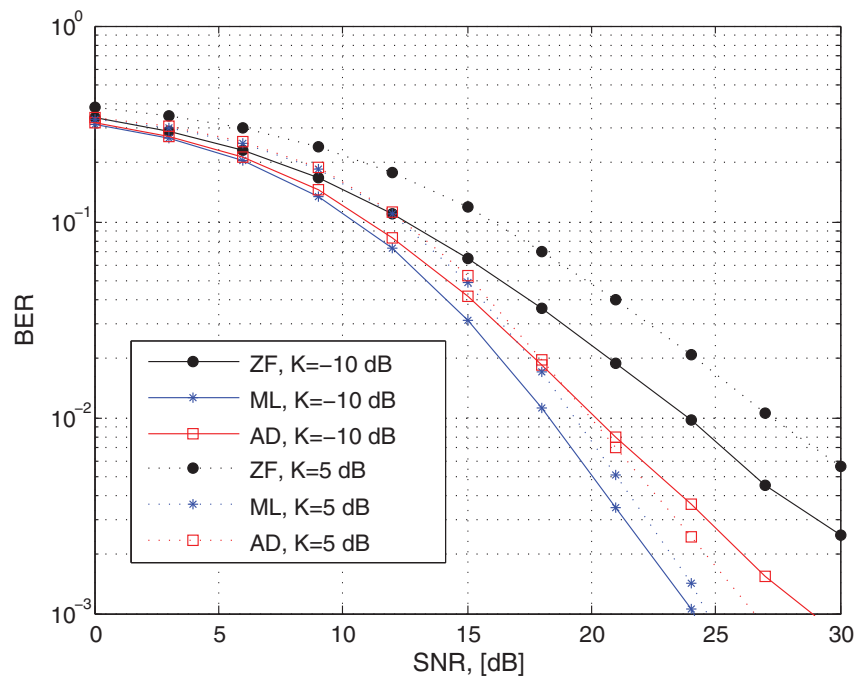

(b) Conventional LR LoS channel.

Fig. 2. BER curves for three different detection strategies based on a 16QAM modulation.

modern practical receivers. Further, when the antenna elements are positioned in such a way to guarantee subchannel orthogonality, the attained performance was shown to be reasonably good, especially for high values of the $K$-factor. On the contrary, the scheme advantages diminish when it is applied to a conventional antenna configuration since the channel matrix is inherently rank deficient and therefore yields a deteriorating performance with the increasing Ricean $K$-factor.

\section{ACKNOWLEDGMENTS}

The authors would like to thank Dr. Ioannis Sarris, formerly of Bristol University, for his valuable help and stimulating technical discussions during the first stage of our work. They would also like to acknowledge the support of the Scottish Funding Council in the Joint Research Institute for Signal and Image Processing with Heriot-Watt University which is a part of the Edinburgh Research Partnership. 
TABLE I

AD PERFORMANCE CHARACTERISTICS FOR TWO DIFFERENT LOS CHANNEL MODELS AS A FUNCTION OF THE $K$-FACTOR.

\begin{tabular}{|c|c|c|c||c|c|c|}
\hline \multirow{2}{*}{$K$-factor } & \multicolumn{2}{|c||}{ Suboptimum HR LoS channel model } & \multicolumn{3}{c|}{ LR LoS channel model } \\
\cline { 2 - 7 } & Analyt. $p_{\kappa}$ & Simul. $p_{\kappa}$ & Compl. reduction & Analyt. $p_{\kappa}$ & Simul. $p_{\kappa}$ & Compl. reduction \\
\hline$K=-10 \mathrm{~dB}$ & 0.2992 & 0.3150 & $26.18 \%$ & 0.2943 & 0.3132 & $25.75 \%$ \\
$K=-5 \mathrm{~dB}$ & 0.3203 & 0.3380 & $28.02 \%$ & 0.2801 & 0.3002 & $24.51 \%$ \\
$K=0 \mathrm{~dB}$ & 0.4372 & 0.4403 & $38.25 \%$ & 0.2052 & 0.2241 & $17.95 \%$ \\
$K=5 \mathrm{~dB}$ & 0.7499 & 0.7501 & $65.61 \%$ & 0.0536 & 0.064 & $4.69 \%$ \\
$K=10 \mathrm{~dB}$ & 0.9526 & 0.9620 & $83.35 \%$ & - & - & - \\
\hline
\end{tabular}

\section{REFERENCES}

[1] I. E. Telatar, "Capacity of multi-antenna Gaussian channels," ATT-Bell Labs Internal Technical Memorandum, June 1995.

[2] G. J. Foschini, "Layered space-time architecture for wireless communications in a fading environment when using multiple antennas," Bell Labs Technical Journal, vol. 1, no. 2, pp. 41-59, Autumn 1996.

[3] R. N. A. Paulraj and D. Gore, Introduction to Space-Time Wireless Communications, Cambridge University Press, U.K., 2003.

[4] U. Fincke and M. Phost, "Improved methods for calculating vectors of short length in a lattice, including a complexity analysis," Mathematics of Computation, vol. 44, pp. 463-471, April 1985.

[5] B. Hassibi and H. Vikalo, "On the sphere-decoding algorithm I. Expected complexity," IEEE Transactions on Signal Processing, vol. 53, no. 8, pp. 2806-2818, August 2005.

[6] J. Jalden and B. Ottersten, "On the complexity of sphere decoding in digital communications," IEEE Transactions on Signal Processing, vol. 53, no. 4, pp. 1474-1484, April 2005.

[7] H. Artes, D. Seethaler, and F. Hlawatsch, "Efficient detection algorithms for MIMO channels: A geometrical approach to approximate ML Detection," IEEE Transactions on Signal Processing, vol. 51, no. 11, pp. 2808-2820, November 2003.

[8] G. Golden, G. J. Foschini, R. Valenzuela, and P. Wolniasky, "Detection algorithm and initial laboratory results using the V-BLAST space-time communication architecture," IEE Electronics Letters, vol. 35, pp. 14$15,1999$.

[9] R. W. Heath and A. J. Paulraj, "Switching between diversity and multiplexing in MIMO systems," IEEE Transactions on Communications, vol. 53, no. 6, pp. 962-968, June 2005.

[10] A. Forenza, M. R. McKay, A. Pandharipande, R. W. Heath, and I. B. Collings, "Adaptive MIMO transmission for exploiting the capacity of spatially correlated channels," IEEE Transactions on Vehicular Technology, vol. 56, no. 2, pp. 619-630, March 2007.

[11] A. Forenza, M. R. McKay, I. B. Collings and R. W. Heath, "Switching between OSTBC and spatial multiplexing with linear receivers in spatially correlated MIMO channels," in Proc. Vehicular Technology Conference (VTC), Melbourne, Australia, May 2006, pp. 1387-1391.
[12] L. Zheng and D. Tse, "Diversity and multiplexing: A fundamental tradeoff in multiple-antenna channels," IEEE Transactions on Information Theory, vol. 49, no. 5, pp. 1073-1096, May 2003.

[13] J. Maurer, G. Matz, and D. Seethaler, "Low-complexity and full-diversity MIMO detection based on condition number thresholding," in Proc. Acoustics, Speech and Signal Processing Conference (ICASSP), vol. 3, Honolulu, Hawaii, April 2007, pp. 61-64.

[14] A. T. James, "Distributions of matrix variates and latent roots derived from normal samples," Ann. Math. Stat., vol. 35, no. 2, pp. 475-501, June 1964.

[15] K. I. Gross and D. S. Richards, "Total positivity, spherical series, and hypergeometric functions of matrix argument," Journal on Approximation Theory, vol. 59, no. 2, pp. 224-246, 1989.

[16] I. S. Gradshteyn and I. M. Ryzhik, Table of Integrals, Series, and Products, Academic Press, Sixth ed., San Diego, 2000.

[17] M. Matthaiou, D. I. Laurenson, and C. -X. Wang, "On analytical derivations of the eigenvalue and condition number distributions of dual non-central Wishart matrices," submitted to IEEE Transactions on Wireless Communications, March 2008.

[18] F. Rashid-Farrokhi, A. Lozano, G. J. Foschini, and R. Valenzuela, "Spectral efficiency of wireless systems with multiple transmit and receive antennas," in Proc. International Symposium on Personal, Indoor and Mobile Radio Communications (PIMRC), vol. 1, London, U.K., September 2000, pp. 373-377.

[19] R. Bohnke, D. Wubben, V. Kuhn, and K. -D. Kammeyer, "Reduced complexity MMSE detection for BLAST architectures," in Proc. Global Telecommunications Conference (GLOBECOM), vol. 4, San Francisco, USA, December 2003, pp. 2258-2262.

[20] I. Sarris and A. R. Nix, "Design and performance assessment of high-capacity MIMO architectures in the presence of a line-of-sight component," IEEE Transactions on Vehicular Technology, vol. 56, no. 4, pp. 2194-2202, July 2007.

[21] M. Matthaiou, D. I. Laurenson, and C. -X. Wang, "Capacity study of vehicle-to-roadside MIMO channels with a line-of-sight component," in Proc. Wireless Communications and Networking Conference (WCNC), Las Vegas, USA, March 2008, pp. 775-779. 